\title{
EOPs: Ejercicios isométricos y su efecto en la presión arterial en pacientes hipertensos
}

\author{
Isometric exercises and their effects on blood pressure in hypertensive patients
}

Matías Castro* y Federico Augustovski **

\begin{abstract}
Resumen
Se presenta brevemente el caso que genera la pregunta. Se confecciona la pregunta en formato "PICO": ¿Los pacientes con diagnóstico de Hipertensión Arterial Esencial que realizan ejercicios isométricos, tienen registros más altos de presión arterial que los que no lo hacen? Se describe la estrategia de búsqueda y sus resultados, y se resume brevemente la revisión sistemática evaluada, que incluyó nueve ensayos clínicos aleatorizados.

La misma concluye que el entrenamiento de resistencia de moderada intensidad no está contraindicado y de confirmarse los resultados, podría ser incluido en las intervenciones para prevenir y tratar la hipertensión. Finalmente, el EOP concluye que a pesar de las debilidades del meta-análisis, se podría permitir la práctica de entrenamientos de resistencia a aquellos que los quieran realizar, y recomendar que los combinen con ejercicios aeróbicos.

\section{Abstract}

The clinical case that generated the question is briefly presented. A question is posed in the PICO format: Do patients with essential hypertension who perform isometric exercise, have higher blood pressure than those who don't perform them? We describe the search strategy and results, and briefly summarize the systematic review evaluated, which included nine randomized trials. It concludes that resistance training of moderate intensity is not contraindicated and, if the results are confirmed, they might be included in interventions to prevent and treat hypertension. Finally, the EOP concluded that despite the weaknesses of the meta-analysis, we could allow the practice of resistance training to those who want to do 'it, and recommend combining them with aerobic exercises.
\end{abstract}

Palabras clave: hipertensión arterial, ejercicio. Key words: hypertension, exercise.

Castro M y Augustovski F. Ejercicios isométricos y su efecto en la presión arterial en pacientes hipertensos. Evid. actual. práct. ambul; 12(1): 31-32. Ene-Mar.2009.

\section{Escenario clínico}

Un paciente de sexo masculino de 37 años de edad, hipertenso esencial sin otro antecedente de relevancia, consulta a su médico de familia para un control periódico de salud. Durante la consulta, el mismo le comenta que desde hace años realiza ejercicios de resistencia, por lo que le pregunta al médico si éstos contribuyen a elevar sus niveles de presión arterial y si le recomienda que los siga realizando o no.

\section{Pregunta que generó el caso}

¿Los pacientes con diagnóstico de Hipertensión Arterial Esencial (población) que realizan ejercicios isométricos (exposición) tienen registros más altos de presión arterial (resultado de interés) que los que no lo hacen (comparación)? Vale destacar que inicialmente se buscaron resultados finales de mayor interés e impacto para el paciente, la salud y el sistema médico como infarto agudo de miocardio, accidente cerebrovascular o mortalidad. Pero debido a que con la estrategia de búsqueda utilizada no se encontraron trabajos que relacionaran dichos resultados con la exposición a evaluar, se terminó optando para formular la pregunta por un resultado intermedio, como lo es el nivel de presión arterial.

\section{Estrategia de búsqueda}

En una primera instancia se realizó una búsqueda en Pubmed limitando el resultado a Meta-Analysis, Randomized Controlled Trial, Review y empleando las palabras claves "Hypertension"[Mesh] AND "Exercise"[Mesh] AND "Myocardial Infarction"[Mesh] AND "Cerebrovascular Disorders"[Mesh]. En la segunda instancia sólo se utilizaron como palabras claves "Hypertension"[Mesh] AND "Exercise"[Mesh]. Se seleccionó una revisión sistemática reciente que parecía responder la pregunta.

\section{Hipertensión arterial y ejercicios de resistencia}

La hipertensión arterial (HTA) es un factor de riesgo mayor de enfermedad cardiovascular (ECV) la que se se ha convertido en la primera causa de riesgo de muerte en el mundo'. La relación de presión arterial y riesgo de eventos de cardiovascu- lares es contínua, consistente e independiente de otros factores de riesgo; implicando la presión arterial alta un mayor riesgo de infarto de miocardio, insuficiencia cardíaca, enfermedad cerebrovascular y renal. Por ejemplo, para los individuos de 40 a 70 años, cada incremento de $20 \mathrm{mmHg}$ en la presión arterial sistólica (PAS) o de $10 \mathrm{mmHg}$ en la diastólica (PAD) duplica el riesgo de ECV en todo el rango que va desde $115 / 75$ hasta $185 / 115 \mathrm{mmHg}^{2}$. Con respecto a la práctica de actividad física, sabemos que los regímenes de ejercicios aeróbicos ejercen un efecto beneficioso en la presión arterial sistólica y pueden reducir la incidencia de hipertensión ${ }^{3-4}$, disminuyendo la presión PAS y la PAD en aproximadamente 5 y $3 \mathrm{mmHg}$ respectivamente ${ }^{5-6}$. Si bien los regímenes de ejercicios de resistencia, de fuerza o isométricos no habían sido considerados tradicionalmente beneficiosos puesto que pueden incrementar la PAS y PAD durante el ejercicio ${ }^{7}$; tanto la Asociación Estadounidense del Corazón (American Heart Association $)^{8}$ como el Colegio Estadounidense de Medicina del Deporte (American College of Sports Medicine) ${ }^{9}$ han aprobado el entrenamiento de resistencia como un complemento para los programas de ejercicios aeróbicos en la prevención, el tratamiento y el control de la hipertensión arterial.

Es por ello que en la práctica médica cotidiana se genera una incógnita sobre qué recomendación o consejo brindar a un paciente hipertenso que realiza o quiere realizarlo, y las implicancias de los ejercicios de resistencia sobre su salud.

En el caso que nos interesa existe la preocupación y la duda sobre el impacto del ejercicio de resistencia como factor independiente en los niveles de presión arterial.

\section{Resumen de la evidencia}

Fuente: Cornelissen $\mathrm{V}$ y col. Effect of resistance training on resting blood pressure: a meta-analysis of randomized controlled trials. J Hypertens. 2005 Feb; 23(2):251-9.

Objetivos: Evaluar el efecto del entrenamiento de resistencia sobre la presión arterial en adultos sedentarios.

Diseño: Meta-análisis de nueve ensayos clínicos controlados aleatorizados.

Búsqueda: Se buscaron artículos a través de la base de datos de MEDLINE (desde 1966 hasta diciembre del 2003), usando

\footnotetext{
* Alumno de la Carrera de Medicina del Instituto Universitario Hospital Italiano

** Servicio de Medicina Familiar y Comunitaria del Hospital Italiano de Buenos Aires. federico.augustovski@ hospitalitaliano.org.ar
} 
como palabras claves 'isometric', 'static', 'resistance', 'strength training', 'weight training' and 'blood pressure'. Solamente los estudios que fueron publicados en revistas con revisión de pares (peer-review) fueron incluídos.

Para la selección de estudios se tuvo en cuenta:

- Población: mayores de 18 años hipertensos o normotensos sin otras enfermedades concomitantes. La mayoría de los participantes fueron de sexo masculino $(61 \%)$ y la mediana de edad fue de 69 años (rango de 20 a 72 años). La mayoría de los estudios fueron realizados sobre población normotensa y solo tres estudios, en pacientes hipertensos.

- Intervenciones: programas de entrenamientos que sólo hubieran incluído ejercicios de resistencia excluyendo ejercicios aeróbicos, con una duración de los programas de al menos cuatro semanas. El entrenamiento de resistencia fue definido como los programas que usan entrenamientos de fuerza, peso, estáticos $\mathrm{y} / \mathrm{o}$ isométricos para aumentar la fuerza, potencia y o resistencia muscular. La mayoría de los estudios incluídos utilizaron entrenamiento dinámico y un estudio utilizó entrenamiento de resistencia estático. La frecuencia media de entrenamiento fue de tres veces por semana y la intensidad osciló en el rango de 30 a $90 \%$ del VO2 máximo. - Resultados: fueron resultados primarios la PAS y la PAD en ambos grupos y resultados secundarios, el consumo máximo de oxigeno, la frecuencia cardíaca y el peso corporal.

\section{Resultados principales}

- Nueve ensayos clínicos controlados aleatorizados (con 12 grupos de estudio) fueron incluidos, con un total de 341 participantes - La tasa promedio de abandono fue del $15 \%$.

- Los gráficos de embudo* para la presión arterial sistólica y la presión arterial diastólica fueron levemente asimétricos con pequeños cambios en la presión arterial en los estudios de mayor duración. Sin embargo, el coeficiente de correlación Kendall Tau* fue no significativo ( $p=0,27$ y $p=0,73$, respectivamente).

- No hubo heterogeneidad ${ }^{*}$ estadísticamente significativa en el meta-análisis de la PAS ni en el de la PAD.

- Cuando se compararon sus resultados con el de los controles y se ponderaron los resultados sobre la base del tamaño de la muestra*, el entrenamiento de resistencia estuvo asociado con una reducción significativa en la PAD $(-3,5 \mathrm{mmHg}$, IC95\% -6,1 a $-0,9)$ sin cambios significativos para la PAS.

- Cuando la ponderación fue aplicada usando la inversa de la varianza del cambio de presión ${ }^{\star}$ el ejercicio de resistencia estuvo asociado con una disminución significativa tanto de la PAS $(-6,0 \mathrm{mmHg}, \mathrm{IC} 95 \%-10,4 \mathrm{a}-1,6)$ como de la PAD $(-4,7 \mathrm{mmHg}$, IC95\% -8.1 a -1.4)

- En el análisis de subgrupos, sólo la duración del seguimiento aparentó estar considerablemente asociada con los cambios de presión arterial. Los estudios con largos seguimientos (mayores a 15 semanas) mostraron cambios más pequeños en la PAS $(p<0,01)$ y en la PAD $(p<0,05)$ que los que duraron menos de 15 semanas.

Conclusión de los autores: El entrenamiento de resistencia de moderada intensidad no está contraindicado y podría ser incluído en las intervenciones para prevenir y tratar la hipertensión. Sin embargo, hacen falta más estudios para confirmarlo.

\section{Comentario y conclusión}

Por un lado el meta-análisis que hemos resumido demostró una reducción significativa de $3,5 \mathrm{mmHg}$ de la $\mathrm{PAD}$ y una reducción no significativa o en el límite de $3,2 \mathrm{mmHg}$ de la PAS; y arfirma que los entrenamientos de resistencia de moderada intensidad (preferentemente combinados con ejercicios aeróbicos) podrían ser incorporados dentro de las intervenciones no farmacológicas para la prevención y el tratamiento de la HTA tomando en cuenta las precauciones apropiadas.

Por otro lado hay que tener en cuenta ciertos aspectos que pueden asumirse como debilidades del estudio como lo son: la búsqueda de información en una sola base de datos electrónica por lo que puede conducir a un sesgo de selección *, la falta de descripción de los métodos usados para seleccionar los estudios y extraer la información, la posibilidad de un sesgo de publicación* y la falta de evaluación de la calidad de los estudios incorporados. Todo lo descripto puede conducir a tornar dificultosa la determinación de la veracidad de la evidencia presentada. Además hay que resaltar que de la selección de estudios sólo tres fueron llevados a cabo sobre una población hipertensa, lo que dificulta la extrapolación de los resultados del metanálisis a la población hipertensa. La evidencia disponible en la actualidad nos inclina a pensar que el entrenamiento de resistencia al menos no conlleva a un incremento en los niveles de presión arterial sino que produciría una disminución de dichos registros. Si bien faltan más estudios para poder recomendar los entrenamientos de resistencia aisladamente, consideramos que tampoco hay evidencia que los contraindique. Teniendo en cuenta esto y los efectos beneficiosos de dicho entrenamiento en otros aspectos de la salud -la densidad ósea ${ }^{10}$, los síntomas de osteoartritis $^{11}$ y el perfil lipídico ${ }^{12}$ - y tomando las precauciones adecuadas de acuerdo a las características individuales del paciente y de la intensidad del ejercicio a realizar, podemos permitir la práctica de entrenamientos de resistencia a aquellos que los quieran realizar como es el caso de nuestro paciente, y recomendar que los combinen con ejercicios aeróbicos.

\section{Referencias}

1. World Health Organization. World health report 2002: Quantifying selected major risks to health - other diet-related risk factors and physical inactivity. http://who.int/whr/2002/chapter4/en/index4.html

2. Lewington S, Clarke R, Qizilbash N, et al. Age-specific relevance of usual blood pressure to vascular mortality: A meta-analysis of individual data for one million adults in 61 prospective studies. Lancet. 2002;360:1903-13

3. Whelton, SP, Chin, A, Xin, X, He, J. Effect of aerobic exercise on blood pressure: A meta-analysis of randomized, controlled trials. Ann Intern Med 2002; 136:493.

4. Stewart, KJ, Bacher, AC, Turner, KL, et al. Effect of exercise on blood pressure in older persons: a randomized controlled trial. Arch Intern Med 2005; 165:756.

5. Whelton, SP, Chin, A, Xin, X, He, J. Effect of aerobic exercise on blood pressure: A meta-analysis of randomized, controlled trials. Ann Intern Med 2002; 136:493.

6. Dickinson, HO, Mason, JM, Nicolson, DJ, et al. Lifestyle interventions to reduce raised blood pressure: a systematic review of randomized controlled trials. J Hypertens $2006 ; 24: 215$.

7. Morales, MC, Coplan, NL, Zabetakis, P, Gleim, GW. Hypertension: The acute and chronic response to exercise (editorial). Am Heart J 1991; 122:264. Shepherd, JT. Circulatory response to exercise in health. Circulation1987; 76(SuppIVI):VI3

8. Pollock ML, Franklin BA, Balady GJ, Chaitman BL, Fleg JL, Fletcher B, Limacher M, Pina IL, Stein RA, Williams M, Bazzarre T. AHA Science Advisory: resistance exercise in individuals with and without cardiovascular disease: benefits, rationale, safety, and prescription: an advisory from the Committee on Exercise, Rehabilitation, and Prevention, Council on Clinical Cardiology, American Heart Association; Position paper endorsed by the American College of Sports Medicine. Circulation. 2000;101:828-833.

9. Pescatello LS, Franklin BA, Fagard R, Farquhar WB, Kelley GA, Ray CA. American College of Sports Medicine position stand. exercise and hypertension. Med Sci Sports Exerc. 2004;36:533-553.

10. Nelson ME, Fiatarone MA, Morganti CM, Trice I, Greenberg RA, Evans WJ. Effects of high-intensity strength training on multiple risk factors for osteoporotic fractures: a randomized controlled trial. JAMA. 1994; 272:1909-1914.

11. Ettinger WH Jr, Burns R, Messier SP, Applegate W, Rejeski WJ, Morgan T, Shumaker S, Berry MJ, O’Toole M, Monu J, Craven T. A randomized trial comparing aerobic exercise and resistance exercise with a health education program in older adults with knee osteoarthritis: the Fitness Arthritis and Seniors Trial (FAST). JAMA. 1997; 277:25-31.

12. Honkola A, Forsen T, Eriksson J. Resistance training improves the metabolic profile in individuals with type 2 diabetes. Acta Diabetol. 1997; 34:245-248. 